\title{
Extensive performance on the antisaccade task does not lead to negative transfer
}

\author{
Gene A. Brewer • Gregory J. Spillers • \\ Brittany McMillan • Nash Unsworth
}

Published online: 7 July 2011

(C) Psychonomic Society, Inc. 2011

\begin{abstract}
Executive-control processes regulate thoughts, emotions, actions, and behaviors that are critical for everyday functioning. Recently, researchers have suggested that these processes can be flexibly modified by tasks that require executive control. Specifically, it has been argued that executive-control tasks can deplete these executive-control processes, which can in turn lead to negative transfer on subsequent task performance. Importantly, the degrees of malleability in executive-control processes and transfer to different tasks are of ongoing debate. The present study critically examined the hypothesis that executive-control processes can be exerted and whether or not this exertion would negatively transfer to performance on various subsequent tasks. Across a series of experiments, negative transfer effects from extensive performance on the antisaccade task were not found. Traditional hypothesis testing and Bayes factor computations were used to validate these findings. Collectively, the present results put in question the use of the antisaccade task to observe both near and far negative transfer from using executive-control processes.
\end{abstract}

Keywords Working memory· Attention

Executive control refers to the set of general-purpose control processes that regulate thought, action, and even emotion. Although executive control can be considered a

G. A. Brewer $(\bowtie)$

Department of Psychology, Arizona State University,

Tempe, AZ 85287-1104, USA

e-mail: gene.brewer@asu.edu

G. J. Spillers $\cdot$ B. McMillan $\cdot$ N. Unsworth Department of Psychology, University of Oregon, Eugene, OR, USA unitary, domain-general construct, previous work has also suggested that executive control represents a family of interrelated processes, including updating, inhibition, and task switching, to name a few (Miyake, Friedman, Emerson, Witzki, Howerter, \& Wager 2000). The extent to which executive-control processes are immutable or whether they can be modified based on prior acts of executive control is emerging as a promising area for researchers. More specifically, decrements in performance on a task that is preceded by another task can be referred to as depletion (Schmeichel, Vohs, \& Baumeister, 2003). Recent work has been concerned with depleting executive-control processes so that they are temporarily weakened for future use (Schmeichel, 2007). Given the consequences of depleting executive control, these concepts have recently been examined in a number of research domains, including developmental, aging, social, clinical, and cognitive psychology, and neuroscience (Muraven \& Baumeister, 2000). The aim of the present study was to test the extent to which implementing executive control to complete the antisaccade task would transfer negatively to other executive-control tasks.

Recent work on depletion effects has focused more on executive-control processes than on general self-regulatory processes (Schmeichel, 2007). In Schmeichel's Experiment 1, participants watched a 6-min video of a woman being interviewed without audio. Unrelated words were presented at the bottom of the screen. The control group simply watched the video, whereas the experimental group was instructed to watch the video and not pay attention to the words. Theoretically, executive control is needed to not look at words, because they tend to automatically capture attention. Thus, inhibiting words for 6 min should deplete executive control. Following the film clip, all participants then performed either an operation or sentence span task. Schmeichel reported that the experimental group scored more poorly on both measures of 
working memory as compared with the control group, concluding that ". . . depleted self-regulatory resources may more precisely be considered instances of reduced resources for executive control" (p. 251; see also Schmeichel et al., 2003). Cohen's $d$ effect size measures in Schmeichel's Experiment 1 ranged from 0.42 to 0.53 across span measures (i.e., medium effect sizes).

Similar results were found by Persson, Welsh, Jonides, and Reuter-Lorenz (2007) examining interference resolution in working memory tasks. Specifically, in their Experiment 1, participants performed a verb-generation task under either high or low interference. Following the verb-generation task, participants performed an item-recognition task. Persson et al. found that the group who performed the verb-generation task under high interference performed more poorly on the item-recognition task than did the low-interference group, suggesting that resolving interference depleted executivecontrol processes such that they could not be used on the subsequent item-recognition task (i.e., near transfer). In their Experiment 2, participants performed a stop-signal task with either few stops or many stops, and then performed the verbgeneration task. Persson et al. hypothesized that the executive-control processes needed in the stop-signal task were different from those needed in the verb-generation task, and thus they did not expect any depletion effects to arise (i.e., far transfer). Consistent with this theorizing, there were no differences between the groups. In a final experiment, participants performed the item-recognition task under either high or low interference and then performed a pairedassociates task with proactive interference. Persson et al. found that performing the high-interference task resulted in poorer subsequent performance (although, technically, this effect was only significant if a one-tailed test was used). Similar to Schmeichel, Persson et al. concluded that executive-control processes "are resource limited and can be temporarily depleted" (p. 1578).

Together, these studies suggest that it may be possible to deplete executive-control processes, resulting in impaired performance on subsequent executive-control tasks. Some work suggests that depletion is a fairly widespread decrement, in that any self- or executive-control task will be impaired (Muraven \& Baumeister, 2000; Schmeichel, 2007), whereas other work seems to suggest that the decrements are fairly specific to certain executive-control processes (Persson et al., 2007). However, it is still not conclusive that executive control can be depleted, leading to negative transfer using all executive-control tasks. Also, it is still not clear which conditions are necessary for demonstrating depletion that will lead to negative transfer. The aim of the present study was to critically examine the notion that executive-control processes can be depleted, leading to negative transfer. To examine this hypothesis, we used the antisaccade task as our primary measure of executive control, given that it has been used in many domains to examine executive-control processes. In the antisaccade task, participants are told to fixate on a central cue; after a variable amount of time, a flashing cue appears either to the right or left of fixation, and participants have to shift their attention and gaze to the opposite side of the screen as quickly and accurately as possible. In this task, there is good deal of conflict between the automatic orienting response and the task goal.

Given the reliance on inhibition of prepotent responses inherent within the antisaccade task, it is no surprise that the task has been used, much like the Stroop (1935) task, in a wide array of clinical and developmental research (Munoz \& Everling, 2004). Furthermore, recent work has found that the antisaccade (but not the prosaccade) task is related to working memory (Unsworth, Schrock, \& Engle, 2004), and the antisaccade correlates with other measures of executive control (e.g., Stroop, stop signal) forming a single factor, and this factor is related to other cognitive constructs (Miyake et al., 2000). ${ }^{1}$ These behavioral results are most likely driven by the common need for maintenance of task goals in working memory (i.e., "look away from the flashing cue"). Clearly, the antisaccade task represents a reliable and valid measure of executive control and can be contrasted with the prosaccade task, which represents automatic orienting of attention. Moreover, the antisaccade task is dependent on neural circuitry that has been consistently implicated with executive-control functions (for a review, see McDowell, Dyckman, Austin, \& Clementz, 2008). Specifically, antisaccade performance generates higher activity levels than prosaccade performance in typical saccadic circuitry (i.e., parietal cortex, frontal eye fields, and supplementary eye fields). McDowell et al. went on to suggest that antisaccade performance relies on an additional, qualitatively different neural circuitry relative to prosaccade performance (i.e., dorsolateral prefrontal cortex, anterior cingulate cortex). This same network is also tapped by other executive-functioning tasks, such as Stroop, operation span, and Raven's Progressive Matrices.

Based on prior theorizing, and the fact that the antisaccade task measures executive-control processes, it should be amenable to negative transfer effects to other tasks that rely on executive control. Experiments 1a-1c tested this hypothesis by providing extensive within-session practice on the antisaccade task and looking for both near

\footnotetext{
${ }^{1}$ Aggregating over multiple experiments conducted in our laboratory at the University of Georgia, we report here the reliability and correlations between antisaccade $(\alpha=.84)$ and Stroop $[r(181)=.22$; $\alpha=.58]$, operation span $[r(331)=.27 ; \alpha=.80]$, and Raven's Advanced Progressive Matrices $[r(331)=.25 ; \alpha=.76]$. These correlations are corrected for unreliability and indicate that, overall, there is significant common variance between antisaccade performance and typical executive-control and general fluid intelligence measures.
} 
and far negative transfer. In order to examine possible negative transfer effects, immediately after completing the saccade task, participants performed either the Stroop (near transfer, Exp. 1a), operation span (medium transfer, Exp. 1b), or Raven's Advanced Progressive Matrices (far transfer, Exp. 1c). According to depletion theories of executive control, performing the antisaccade task should lead to depletion on all three tasks, but performing the prosaccade task should not. Also, a no-saccade control condition was present in each experiment to assess whether simply doing any task would lead to negative transfer.

\section{Experiments 1a-1c}

Participants performed 750 trials of a task that required either automatic orienting of attention (prosaccade) or executive control to prevent automatic attentional capture (antisaccade). Importantly, the two tasks differed in their needs for frontally mediated processes (i.e., executive control; McDowell et al., 2008). Prior theorizing has suggested that depletion effects should only occur when executive-control processes are recruited and should not occur when automatic processes drive performance (Schmeichel, 2007). This reasoning suggests that extensive within-session practice on the antisaccade task should deplete executive-control processes, but within-session practice on the prosaccade task should not.

In addition to differing theoretically in the need for executive control, the prosaccade and antisaccade tasks were used in these experiments because measures of performance (accuracy and reaction time) could be obtained from them. Prior work on depletion effects has typically relied only on self-report measures of difficulty to assess depletion on the first task (e.g., Schmeichel, 2007). While self-reports of difficulty certainly provide experiential information regarding the nature of the task, we would prefer to see performance differences between the tasks (differences in both accuracy and latency). It should be noted that we decided to have participants perform 750 trials on the saccade tasks to ensure the possibility of depletion. Previous work has suggested that 6 min of viewing a video clip is sufficient to induce depletion effects, so performing 750 trials on an antisaccade task (roughly $40 \mathrm{~min}$ ) should be enough to deplete executivecontrol processes.

\section{Method}

Participants and design

The participants were a total of 275 undergraduate students recruited from the participant pool at the University of
Georgia. All participants were randomly assigned to ensure that baseline differences in executive control would be equally distributed across conditions. In Experiment 1a, 96 participants were equally assigned to the antisaccade, prosaccade, and control conditions. In Experiment 1b, 33 participants were assigned to the antisaccade condition, 35 to the prosaccade condition, and 34 to the control conditions. In Experiment 1c, 25 participants were assigned to the antisaccade condition, 26 to the prosaccade condition, and 26 to the control condition.

\section{Materials and procedure}

In the beginning of the experiment, all participants in the saccade conditions performed 750 trials on either the prosaccade or antisaccade task. Immediately following the saccade task, participants performed one of the transfer tasks. In Experiment 1a, participants performed the Stroop (1935) task, in $1 \mathrm{~b}$ the operation span task, and in 1c Raven's Advanced Progressive Matrices. Participants in the control conditions for each experiment simply completed the transfer task with no delay and without completing any saccade trials.

\section{Depletion-and-nondepletion tasks: saccade tasks}

Participants stared at a fixation point that was onscreen for a variable amount of time (200-2,200 ms). A white "=" sign was then flashed either to the left or right of fixation (at $11.33^{\circ}$ of visual angle) for $100 \mathrm{~ms}$. This was followed by a 50-ms blank screen and a second appearance of the cue for $100 \mathrm{~ms}$, making it appear as though the cue ("=") repeatedly flashed onscreen. Following another 50-ms blank screen, the target stimulus (a B, P, or R) appeared on screen for $100 \mathrm{~ms}$, followed by masking stimuli (an $\mathrm{H}$ for $50 \mathrm{~ms}$ and an " 8 " that remained onscreen until a response was given). The participants' task was to identify the target letter by pressing a key for B, P, or R (the keys 1 , 2 , or 3) as quickly and accurately as possible. In the prosaccade condition, the flashing cue ("=") and the target appeared in the same location. In the antisaccade condition, the target appeared in the location opposite from the flashing cue. The stimuli were spaced far apart to provide a strong incentive for participants to use the cue. Participants received, in order, 10 practice trials to learn the response mapping, 15 practice trials of that particular saccade task (either pro- or antisaccade), and 750 real trials of that particular saccade task.

Transfer tasks

Stroop (Exp. 1a) Participants were presented with a color word ("red," "green," or "blue") presented in one of three different font colors (red, green, or blue). The participants' 
task was to indicate the font color via keypress (red $=1$, green $=2$, blue $=3$ ). Participants were told to press the corresponding key as quickly and accurately as possible. Participants received 15 trials of response-mapping practice and 6 trials of practice with the real task. They then received two blocks of 75 trials each. In the first block, approximately $50 \%$ of the trials were congruent, such that the word and font color matched (i.e., "red" printed in red), and the other $50 \%$ were incongruent (i.e., "red" printed in green). On the second block of trials, $67 \%$ of the trials were congruent and $33 \%$ were incongruent. We manipulated proportion congruency in this way because prior work had suggested that when there are more congruent than incongruent trials, active goal maintenance is more difficult, and thus, a high proportion of congruent trials likely places a premium on executive-control processes (Kane \& Engle, 2003). For both blocks of trials, the dependent variable was the reaction time difference between incongruent and congruent trials.

Operation span (Exp. 1b) Participants solved a series of math operations while trying to remember a set of unrelated letters. After solving each math operation, participants were presented with a letter for $1 \mathrm{~s}$. Immediately after the letter was presented, the next operation was presented. Three presentations of each list length (3-7) were presented, for a total of 75 trials. The order of list length varied randomly. At recall, letters from the current set were to be recalled in the correct order by clicking on the appropriate letters. Participants received three sets of practice, and the order of set sizes, math operations, and sequences of letters varied across participants. The dependent variable was the proportion of correct items recalled in the correct position.

Raven's advanced progressive matrices (Exp. 1c) The Raven is a measure of abstract reasoning and general fluid intelligence. The test consists of 18 items presented in ascending order of difficulty (easiest to hardest). Each item consists of a display of $3 \times 3$ matrices of geometric patterns with the bottom right pattern missing. The task for the participant was to select from among eight alternatives the one that correctly completed the overall series of patterns. Participants had $10 \mathrm{~min}$ to complete the 18 items. A participant's score was the total number of correct solutions.

\section{Results}

Depletion and nondepletion tasks

As shown in Table 1, across all experiments performance on the prosaccade task was more accurate and correct reaction
Table 1 Accuracy (Acc) and correct reaction time (RT) as a function of saccade task and Experiment

\begin{tabular}{llllll}
\hline & \multicolumn{2}{l}{ Prosaccade } & & \multicolumn{2}{l}{ Antisaccade } \\
\cline { 2 - 3 } \cline { 5 - 6 } & Acc & RT & & Acc & RT \\
\hline Experiment 1a & .90 & 603 & & .64 & 682 \\
Experiment 1b & .92 & 585 & & .63 & 683 \\
Experiment 1c & .89 & 540 & & .57 & 699 \\
\hline
\end{tabular}

times were shorter than on the antisaccade task, all $t \mathrm{~s}>2.18$, $p \mathrm{~s}<.05$. As expected, performance was poorer on the antisaccade than on the prosaccade task. This result is consistent with the notion that the antisaccade task was more difficult than the prosaccade task due to the increased need for executive control to avoid looking at the flashing cue. ${ }^{2}$

Transfer tasks

Based on the clear differences between the saccade tasks, we next examined whether performing the antisaccade task would lead to negative transfer effects, as compared with the prosaccade task, on other tasks thought to rely on executive control. In order to assess for negative transfer, we compared performance on the transfer tasks as a function of which saccade task was performed (pro- or antisaccade). Shown in Table 2 are the scores on each transfer task as a function of which saccade task preceded them, as well as the differences between the transfer tasks as a function of saccade task. Also shown in Table 2 are the associated $t$ and $p$ values and $95 \%$ confidence intervals for the critical test of group differences, as well as the associated Bayes factors based on Rouder, Speckman, Sun, Morey, and Iverson (2009; BF1, with a scale $r$ on effect size $=1$ ) and Gallistel (2009; BF2), respectively.

\footnotetext{
$\overline{2}$ The participants might not have been moving their visual attention in accordance with the antisaccade instructions, but might instead have relied on some other strategy to raise their performance above mathematical chance (i.e., 33\%). To address this issue, we conducted an additional study $(n=20)$ that matched the antisaccade condition in every way, except for one critical change: That is, we removed the antisaccade cue that was indicative of the side of the screen on which the target (B, P, or R) would occur. Therefore, any strategies other than shifting visual attention to the cued location could still be used to raise accuracy above three-alternative forced choice chance performance. If performance was still less than that found in the antisaccade experiments $(M=.61)$, then one could infer that participants were directing their visual attention in a manner consistent with the task instructions (i.e., "look away from the cue"). Accuracy in the additional, no-cue saccade task $(M=.43, S E=.02)$ was significantly lower than accuracy in the antisaccade experiments, $t(172)=13.98, p<.001, d=2.13$. These results indicate that participants in the antisaccade conditions of Experiments 1a, 1b, and 1c were using executive control to direct their visual attention to the location opposite the cue rather than only relying on some other strategy to complete the task.
} 
Table 2 Performance on the transfer tasks as a function of saccade task

\begin{tabular}{llllllllll}
\hline Measure & Control & Prosaccade & Antisaccade & Diff & $t$ & $p$ & BF1 & BF2 & $95 \%$ C.I. \\
\hline Stroop 50-50 & 99 & 127 & 117 & 10 & 0.65 & .52 & 4.37 & 6.19 & $(-42.47,21.71)$ \\
Stroop 67-33 & 172 & 177 & 184 & 7 & 0.22 & .83 & 5.18 & 9.02 & $(-56.49,70.30)$ \\
Ospan & 61.56 & 62.49 & 62.79 & 0.30 & 0.14 & .89 & 5.39 & 8.55 & $(-4.61,4.00)$ \\
Raven & 10.38 & 9.68 & 10.12 & 0.44 & 0.54 & .59 & 4.20 & 5.13 & $(-2.06,1.19)$ \\
\hline
\end{tabular}

Stroop 50-50 (67-33), Stroop task with 50\% (67\%) congruent trials; Ospan, operation span task; Raven, Raven's Advanced Progressive Matrices; Control, score with no saccade task before completing transfer task; Prosaccade, score with 750 prosaccade trials before completing the transfer task; Antisaccade, score with 750 antisaccade trials before completing the transfer task; Diff, absolute difference between saccade conditions; $t, t$ value for the difference between the saccade conditions; $p$, corresponding $p$ value; BF1, Bayes factor from Rouder et al. (2009); BF2, Bayes factor from Gallistel (2009); 95\% C.I., 95\% confidence interval around the absolute difference between saccade conditions

Bayes factors provide a means of inferring evidence in favor of the null hypothesis over evidence in favor of the alternative. That is, Bayes factors represent the odds ratio of evidence in favor of one hypothesis (i.e., in this case, no negative transfer from extensive antisaccade performance) over the alternative (i.e., negative transfer from extensive antisaccade performance). To preface the results, there were no differences between the control conditions and the saccade conditions in any experiment. Therefore, we report the one-way ANOVA in each case and then compare antisaccade to prosaccade performance directly.

Experiment 1a: stroop For the 50-50 Stroop condition, there was no overall difference between conditions, $p>.30$. Inconsistent with prior negative-transfer research, the specific effect of saccade condition was not significant, $p>$.64. Although all groups demonstrated Stroop effects, there was no difference between the groups in terms of the magnitudes of the Stroop effects. Furthermore, both Bayes factors suggested that the odds were greater than $4: 1$ in favor of no difference between pro- and antisaccade conditions.

Similar to the 50-50 condition, the 67-33 condition also did not demonstrate an overall effect of condition or difference between saccade conditions, $p>.91$ and .82 , respectively. Furthermore, both Bayes factors suggested that the odds were greater than 5:1 in favor of the null hypothesis. Consistent with previous research (Kane \& Engle, 2003), there was a significant effect of Stroop condition, suggesting that the Stroop effect was $58 \mathrm{~ms}$ larger in the 67-33 condition than in the 50-50 condition, $t(95)=6.02, p<.001$.

Experiment 1b: operation span For the operation span task, there was no overall effect of condition or difference between saccade conditions on the total numbers of letters recalled in the correct positions, $p>.86$ and .88 , respectively. Furthermore, both Bayes factors suggested that the odds were greater than 5:1 in favor of no difference between proand antisaccade conditions.
Experiment 1c: raven's advanced progressive matrices For the Raven matrices, there was no overall effect of condition or difference between saccade conditions on the total number of letters recalled in the correct position, $p>.66$ and .58, respectively. Furthermore, both Bayes factors suggested that the odds were greater than 4:1 in favor of no difference between pro- and antisaccade conditions.

\section{Discussion}

Experiments 1a-1c examined whether extensive withinsession practice on an executive-control task (antisaccade) would transfer negatively to other tasks thought to share executive-control processes. Participants performed either an automatic orienting task (prosaccade) or a task that required executive control of attention to prevent automatic attentional capture (antisaccade). Consistent with prior work on the pro- and antisaccade tasks, accuracy was higher and correct reaction times were shorter on the prosaccade than on the antisaccade task. In terms of transfer, the results suggested that there was virtually no evidence for negative transfer on any task. Specifically, in all cases, differences between the groups on the transfer tasks failed to reach conventional levels of statistical significance, and the estimated Bayes factors suggested that the odds were always more than 4:1 in favor of no effect of transfer from depleting executive control. Thus, of the four possible transfer effects that could have been found, none were found in the present study, and the evidence was by and large consistent with the null hypothesis. These results cast serious doubt on the generality of negative transfer from depletion of executive-control processes.

Prior theorizing (e.g., Schmeichel, 2007) suggested that within-session practice on an executive-control task (antisaccade) should deplete executive-control processes such that subsequent performance on another executive-control task should be harmed. Within-session practice on a relatively automatic (prosaccade) task, however, should not influence task performance on subsequent tasks. Across 
all three experiments, prosaccade performance was more accurate and prosaccade reaction times were shorter than with the antisaccade task. In terms of transfer, however, there was no evidence for negative transfer on any of the tasks. Specifically, the prosaccade and antisaccade groups performed nearly equivalently on all four measures of transfer, and none of these conditions differed significantly from a control group who performed no saccade task. These results are inconsistent with recent claims that extensive within-session practice depletes executive-control resources, leading to negative transfer on other executive-control and cognitive-ability measures.

Yet, at the same time, there was evidence in favor of the alternative in several cases, independently of group differences. For instance, we replicated the effect of proportional congruence on the Stroop effect (Kane \& Engle, 2003). Using Rouder et al.'s (2009) calculation, the Bayes factor for these effects is .004 in Experiment 1a, suggesting that the odds were more than 10:1 in favor of the alternative hypothesis (i.e., larger Stroop effects in the 67\%-33\% condition). Thus, despite the fact that there was only about a 53-ms difference in the magnitude of the Stroop effect across proportional congruence conditions, this effect was highly robust, and we can be fairly confident that it was in fact a true effect. Therefore, the use of Bayesian inference allows us to examine evidence in favor of the null hypothesis over evidence in favor of the alternative hypothesis. In all cases, the evidence indicated that it was more likely that there was no effect of depletion on any of the measures. Like Rouder et al. (2009) and Gallistel (2009), we think that it is important to examine null effects and to further determine whether performance on executive-control measures is invariant across putative depletion manipulations.

One possible alternative explanation to our results is that we simply did not deplete our participants, and thus negative transfer could not be demonstrated. We find this argument untenable, given that our participants performed 750 antisaccade trials (over about $40 \mathrm{~min}$ ) prior to performing a subsequent executive-control task. Furthermore, recall that Schmeichel (2007; Schmeichel et al., 2003) had participants simply watch a video and ignore words onscreen for $6 \mathrm{~min}$. Surely if watching a video for $6 \mathrm{~min}$ can readily deplete one's resources to the point that their subsequent performance is harmed, then performing an antisaccade task (which is quite demanding) over $40 \mathrm{~min}$ should cause as much, if not more, depletion, leading to similar performance deficits. Alternatively, one reviewer suggested that participants might have automatized the antisaccade task by the 750th trial. However, recent research has suggested that it takes more than double that number trials before the antisaccade can be performed in an automatic fashion (Unsworth, Spillers, Brewer, \& McMillan, 2011). Still, it is a tantalizing notion that shorter performance intervals may yield larger depletion effects. Therefore, based on prior research, our depletion manipulation should have been effective in depleting executive-control processes.

Another potential issue with the use of the antisaccade task in the present study is that perhaps in order to demonstrate depletion effects, the task must engage multiple executivecontrol processes. On the one hand, the antisaccade task primarily engages goal maintenance and inhibitory processes (Munoz \& Everling, 2004; Unsworth et al., 2004), and the possibility exists that it simply did not engage enough different control processes to lead to negative transfer. On the other hand, this argument predicts that performance on the Stroop task (near transfer) should be more susceptible to depletion than performance on Raven's matrices (far transfer). Importantly, there was limited evidence for the near/far transfer hypothesis in the data. Thus, future work will be needed to better determine whether multiple executive-control processes need to be engaged to allow for training and depletion, or whether a single executivecontrol process can be fatigued, leading to transfer.

The present results suggest no evidence for depletion of executive control in terms of negative transfer from performing the antisaccade task. The boundary conditions on depleting executive-control processes leading to negative transfer are far from being completely specified. Future work is needed to critically examine these notions and to delineate the conditions under which it may be possible to find negative transfer. Furthermore, future work is needed to critically examine whether these effects actually represent true depletion effects, or whether other variables are at play. Overall, the results of the present study cast doubt on the generality of depleting executive control.

Author note We thank Eric Ruthruff and Rob McCann for comments on a previous version of the manuscript.

\section{References}

Gallistel, C. R. (2009). The importance of proving the null. Psychological Review, 116, 439-453. doi:10.1037/a0015251

Kane, M. J., \& Engle, R. W. (2003). Working-memory capacity and the control of attention: The contributions of goal neglect, response competition, and task set to Stroop interference. Journal of Experimental Psychology: General, 132, 47-70. doi:10.1037/ 0096-3445.132.1.47

McDowell, J. E., Dyckman, K. A., Austin, B. P., \& Clementz, B. A. (2008). Neurophysiology and neuroanatomy of reflexive and volitional saccades: Evidence from studies of humans. Brain and Cognition, 68, 255-270. doi:10.1016/j.bandc.2008.08.016

Miyake, A., Friedman, N. P., Emerson, M. J., Witzki, A. H., Howerter, A., \& Wager, T. D. (2000). The unity and diversity of executive functions and their contributions to complex "frontal lobe" tasks: A latent variable analysis. Cognitive Psychology, 41, 49-100. doi:10.1006/cogp.1999.0734

Munoz, D. P., \& Everling, S. (2004). Look away: The anti-saccade task and the voluntary control of eye movement. Nature Reviews Neuroscience, 5, 218-228. doi:10.1038/nrn1345 
Muraven, M., \& Baumeister, R. F. (2000). Self-regulation and depletion of limited resources: Does self-control resemble a muscle? Psychological Bulletin, 126, 247-259. doi:10.1037/ 0033-2909.126.2.247

Persson, J., Welsh, K. M., Jonides, J., \& Reuter-Lorenz, P. A. (2007). Cognitive fatigue of executive processes: Interaction between interference resolution tasks. Neuropsychologia, 45, 1571-1579. doi:10.1016/j.neuropsychologia.2006.12.007

Rouder, J. N., Speckman, P. L., Sun, D., Morey, R. D., \& Iverson, G. (2009). Bayesian $t$ tests for accepting and rejecting the null hypothesis. Psychonomic Bulletin \& Review, 16, 225-237. doi:10.3758/PBR.16.2.225

Schmeichel, B. J. (2007). Attention control, memory updating, and emotion regulation temporarily reduce the capacity for executive control. Journal of Experimental Psychology: General, 136, 241-255. doi:10.1037/0096-3445.136.2.241
Schmeichel, B. J., Vohs, K. D., \& Baumeister, R. F. (2003). Intellectual performance and ego depletion: Role of the self in logical reasoning and other information processing. Journal of Personality and Social Psychology, 85, 33-46. doi:10.1037/0022-3514.85.1.33

Stroop, J. R. (1935). Studies of interference in serial verbal reactions. Journal of Experimental Psychology, 18, 643-662. doi:10.1037/ 0096-3445.121.1.15

Unsworth, N., Schrock, J. C., \& Engle, R. W. (2004). Working memory capacity and the antisaccade task: Individual differences in voluntary saccade control. Journal of Experimental Psychology: Learning, Memory, and Cognition, 30, 1302-1321. doi:10.1037/ 0278-7393.30.6.1302

Unsworth, N., Spillers, G. J., Brewer, G. A., \& McMillan, B. (2011). Attention control and the antisaccade task: A response time distribution analysis. Acta Psychologica, 137, 90-100. doi:10.1016/j.actpsy.2011.03.004 